\title{
ZOOTAXA
}

\section{Nomenclatural Studies Toward a World List of Diptera Genus-Group Names. Part II: Camillo Rondani}

\author{
JAMES E. O'HARA ${ }^{1}$, PIERFILIPPO CERRETTI ${ }^{2}$, THOMAS PAPE $^{3}$ \\ $\&$ NEAL L. EVENHUIS ${ }^{4}$
}

1. Canadian National Collection of Insects, Agriculture and Agri-Food Canada, 960 Carling Avenue, Ottawa, Ontario, K1A 0C6, Canada; email: james.ohara@agr.gc.ca

2. Centro Nazionale Biodiversità Forestale "Bosco Fontana", Corpo Forestale dello Stato, Via C. Ederle 16/A, 37100 Verona, Italy; email:pierfilippocerretti@yahoo.it

3. Natural History Museum of Denmark, Universitetsparken 15, 2100 Copenhagen, Denmark; email: TPape@snm.ku.dk

4. J. Linsley Gressitt Center for Entomological Research, Bishop Museum, 1525 Bernice Street, Honolulu, Hawaii 96817-2704, USA; email: NealE@bishopmuseum.org

\section{6}

Magnolia Press

Auckland, New Zealand 
Nomenclatural Studies Toward a World List of Diptera Genus-Group Names. Part II: Camillo Rondani JAMES E. O'HARA, PIERFILIPPO CERRETTI, THOMAS PAPE \& NEAL L. EVENHUIS

268 pp.; $30 \mathrm{~cm}$.

23 Dec. 2011

ISBN 978-1-86977-843-9 (paperback)

ISBN 978-1-86977-844-6 (Online edition)

\section{FIRST PUBLISHED IN 2011 BY}

Magnolia Press

P.O. Box 41-383

Auckland 1346

New Zealand

e-mail: zootaxa@mapress.com

http://www.mapress.com/zootaxa/

(C) 2011 Magnolia Press

All rights reserved.

No part of this publication may be reproduced, stored, transmitted or disseminated, in any form, or by any means, without prior written permission from the publisher, to whom all requests to reproduce copyright material should be directed in writing.

This authorization does not extend to any other kind of copying, by any means, in any form, and for any purpose other than private research use.

ISSN 1175-5326 (Print edition)

ISSN 1175-5334 (Online edition) 


\section{Table of contents}

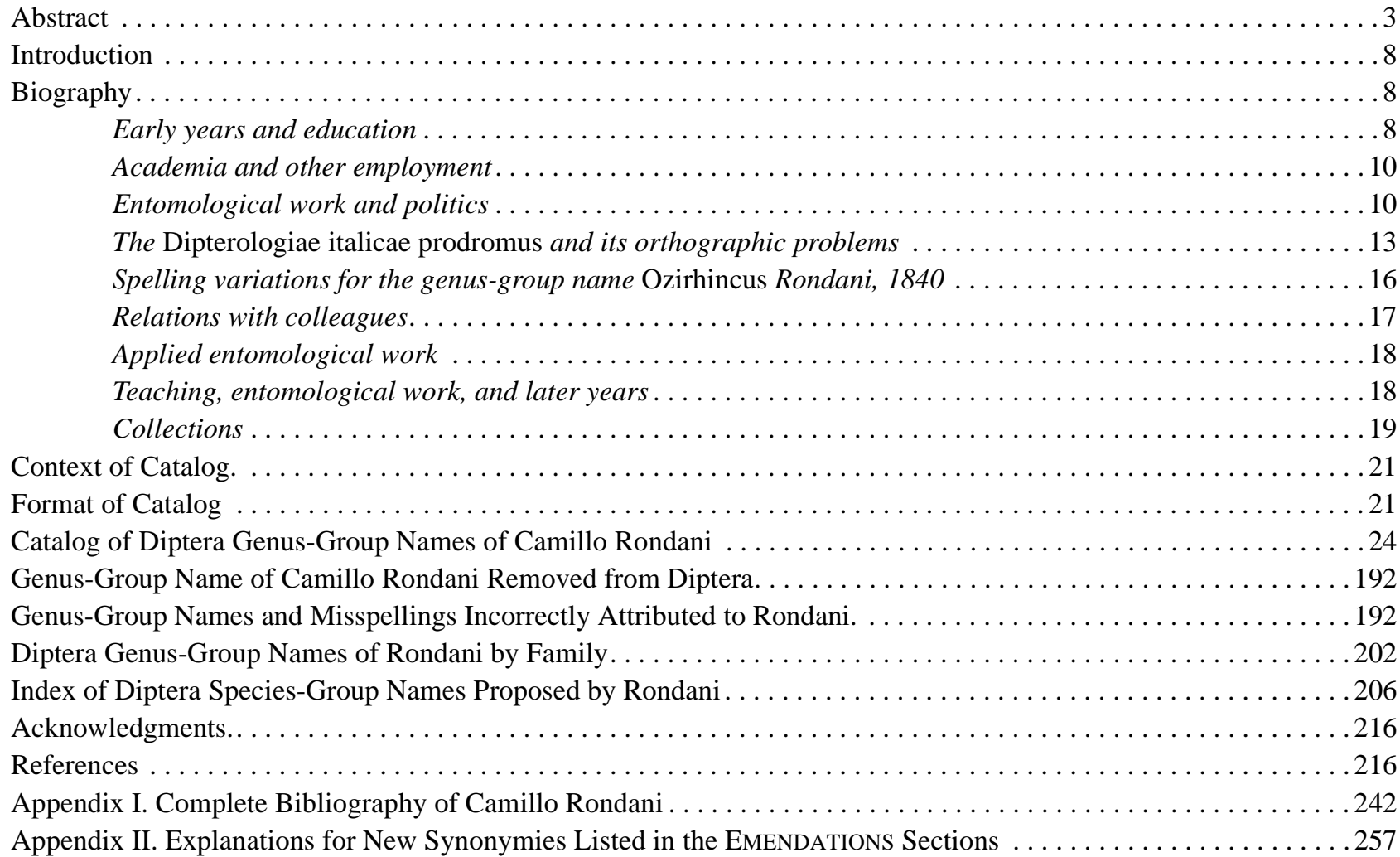

\section{Abstract}

The Diptera genus-group names of Camillo Rondani are reviewed and annotated. A total of 601 nomenclaturally available genus-group names in 82 families of Diptera are listed alphabetically. For each name the following are given: author, year and page of original publication, originally included species [and first included species if none were originally included], type species and method of fixation, current status of the name, family placement, and a list of any emendations of it that have been found in the literature. Remarks are given to clarify nomenclatural or taxonomic information. In addition, an index is provided to all the species-group names of Diptera proposed by Rondani $(1,236$, of which 1,183 are available) with bibliographic reference to each original citation. Appended to this study is a full bibliography of Rondani's works and a list with explanations for all new synonymies arising from revised emendations.

Corrected or clarified type-species and/or corrected or clarified type-species designations are given for the following genus-group names: Anoplomerus Rondani, 1856 [Dolichopodidae]; Biomya Rondani, 1856 [Tachinidae]; Bremia Rondani, 1861 [Cecidomyiidae]; Deximorpha Rondani, 1856 [Tachinidae]; Elasmocera Rondani, 1845 [Asilidae]; Enteromyza Rondani, 1857 [Oestridae]; Exogaster Rondani, 1856 [Tachinidae]; Istocheta Rondani, 1859 [Tachinidae]; Istoglossa Rondani, 1856 [Tachinidae]; Lejogaster Rondani, 1857 [Syrphidae]; Lignodesia Rondani, 1868 [Phaeomyiidae]; Medorilla Rondani, 1856 [Tachinidae]; Meroplius Rondani, 1874 [Sepsidae]; Nodicornis Rondani, 1843 [Dolichopodidae]; Omalostoma Rondani, 1862 [Tachinidae]; Opegiocera Rondani, 1845 [Asilidae]; Petagnia Rondani, 1856 [Tachinidae]; Phaniosoma Rondani, 1856 [Tachinidae]; Proboscina Rondani, 1856 [Tachinidae]; Pyragrura Rondani, 1861 [Tachinidae]; Stemonocera Rondani, 1870 [Tephritidae]; Telejoneura Rondani, 1863 [Asilidae]; Tricoliga Rondani, 1856 [Tachinidae].

The following genus-group names previously treated as available were found to be unavailable: Bombyliosoma Verrall, 1882, n. stat. [Bombyliidae]; Bombylosoma Marschall, 1873, n. stat. [Bombyliidae]; Brachynevra Agassiz, 1846, n. stat. [Cecidomyiidae]; Calliprobola Rondani, 1856, n. stat. [Syrphidae]; Camponeura Verrall, 1882, n. stat. [Syrphidae]; Chlorosoma Verrall, 1882, n. stat. [Stratiomyidae]; Engyzops Verrall, 1882, n. stat. [Calliphoridae]; Exodonta Verrall, 1882, n. stat. [Stratiomyidae]; Histochaeta Verrall, 1882, n. stat. [Tachinidae]; Histoglossa Verrall, 1882, n. stat. [Tachinidae]; Homalostoma Verrall, 1882, n. stat. [Tachinidae]; Hoplacantha Verrall, 1882, n. stat. [Stratiomyidae]; Hoplodonta Verrall, 1882, n. stat. [Stratiomyidae]; Liota Verrall, 1882, n. stat. [Syrphidae]; Lomatacantha Verrall, 1882, n. stat. [Tachinidae]; Machaera Mik, 1890, n. stat. [Tachinidae]; Machaira Brauer \& Bergenstamm, 1889, n. stat. [Tachinidae]; Myiatropa Verrall, 1882, n. stat. [Syrphidae]; Oplacantha Verrall, 1882, n. stat. [Stratiomyidae]. 
Previous First Reviser actions for multiple original spellings missed by previous authors include: Genus-group names-Achanthipodus Rondani, 1856 [Dolichopodidae]; Argyrospila Rondani, 1856 [Bombyliidae]; Botria Rondani, 1856 [Tachinidae]; Chetoliga Rondani, 1856 [Tachinidae]; Chrysoclamys Rondani, 1856 [Syrphidae]; Cyrtophloeba Rondani, 1856 [Tachinidae]; Istocheta Rondani, 1859 [Tachinidae]; Macherea Rondani, 1859 [Tachinidae]; Macronychia Rondani, 1859 [Sarcophagidae]; Pachylomera Rondani, 1856 [Psilidae]; Peratochetus Rondani, 1856 [Clusiidae]; Phytophaga Rondani, 1840 [Cecidomyiidae]; Spylosia Rondani, 1856 [Tachinidae]; Thlipsogaster Rondani, 1863 [Bombyliidae]; Tricogena Rondani, 1856 [Rhinophoridae]; Tricoliga Rondani, 1856 [Tachinidae]; Viviania Rondani, 1861 [Tachinidae]. Species-group name-Sphixapata albifrons Rondani, 1859 [Sarcophagidae].

Acting as First Reviser, the following correct original spellings for multiple original spellings are selected by us: Bellardia Rondani, 1863 [Tabanidae]; Chetoptilia Rondani, 1862 [Tachinidae]; Chetylia Rondani, 1861 [Tachinidae]; Clytiomyia Rondani, 1862 [Tachinidae]; Cryptopalpus Rondani, 1850 [Tachinidae]; Diatomineura Rondani, 1863 [Tabanidae]; Enteromyza Rondani, 1857 [Oestridae]; Esenbeckia Rondani, 1863 [Tabanidae]; Hammomyia Rondani, 1877 [Anthomyiidae]; Hydrothaea Rondani, 1856 [Muscidae]; Hyrmophlaeba Rondani, 1863 [Nemestrinidae]; Limnomya Rondani, 1861 [Limoniidae]; Lyoneura Rondani, 1856 [Psychodidae]; Micetoica Rondani, 1861 [Anisopodidae]; Miennis Rondani, 1869 [Ulidiidae]; Mycetomiza Rondani, 1861 [Mycetophilidae]; Mycosia Rondani, 1861 [Mycetophilidae]; Mycozetaea Rondani, 1861 [Mycetophilidae]; Piotepalpus Rondani, 1856 [Mycetophilidae]; Prothechus Rondani, 1856 [Pipunculidae]; Spyloptera Rondani, 1856 [Limoniidae]; Teremya Rondani, 1875 [Lonchaeidae]; Thricogena Rondani, 1859 [Tachinidae]; Trichopalpus Rondani, 1856 [Scathophagidae]; Trichopeza Rondani, 1856 [Brachystomatidae]; Tricophthicus Rondani, 1861 [Muscidae]; Triphleba Rondani, 1856 [Phoridae]; Xiloteja Rondani, 1863 [Syrphidae].

The following names are new synonymies of their respective senior synonyms: Genus-group names-Acanthipodus Bigot, 1890 of Poecilobothrus Mik, 1878, n. syn. [Dolichopodidae]; Acanthiptera Rondani, 1877 of Achanthiptera Rondani, 1856, n. syn. [Muscidae]; Achantiptera Schiner, 1864 of Achanthiptera Rondani, 1856, n. syn. [Muscidae]; Acydia Rondani, 1870 of Acidia Robineau-Desvoidy, 1830, n. syn. [Tephritidae]; Acyura Rondani, 1863 of Aciura Robineau-Desvoidy, 1830, n. syn. [Tephritidae]; Agaromyia Marschall, 1873 of Agaromya Rondani, 1861, n. syn. [Mycetophilidae]; Ammomyia Mik, 1883 of Leucophora Robineau-Desvoidy, 1830, n. syn. [Anthomyiidae]; Anomoja Rondani, 1871 of Anomoia Walker, 1835, n. syn. [Tephritidae]; Anthracomyia Rondani, 1868 of Morinia RobineauDesvoidy, 1830, n. syn. [Calliphoridae]; Antracomya Lioy, 1864 of Morinia Robineau-Desvoidy, 1830, n. syn. [Calliphoridae]; Anthoeca Bezzi, 1906 of Solieria Robineau-Desvoidy, 1849, n. syn. [Tachinidae]; Antomyza Rondani, 1866 of Anthomyza Fallén, 1810, n. syn. [Anthomyzidae]; Antracia Rondani, 1862 of Nyctia Robineau-Desvoidy, 1830, n. syn. [Sarcophagidae]; Aporomyia Schiner, 1861 of Lypha Robineau-Desvoidy, 1830, n. syn. [Tachinidae]; Asphondilia Rondani, 1861 of Asphondylia Loew, 1850, n. syn. [Cecidomyiidae]; Asteja Rondani, 1856 of Asteia Meigen, 1830, n. syn. [Asteiidae]; Astenia Rondani, 1856 of Blepharicera Macquart, 1843, n. syn. [Blephariceridae]; Astilium Costa, 1866 of Senobasis Macquart, 1838, n. syn. [Asilidae]; Ateleneura Agassiz, 1846 of Atelenevra Macquart, 1834, n. syn. [Pipunculidae]; Athomogaster Rondani, 1866 of Azelia Robineau-Desvoidy, 1830 , n. syn. [Muscidae]; Axista Rondani, 1856 of Axysta Haliday, 1839, n. syn. [Ephydridae]; Bigonichaeta Schiner, 1864 of Triarthria Stephens, 1829, n. syn. [Tachinidae]; Billea Rondani, 1862 of Billaea Robineau-Desvoidy, 1830, n. syn. [Tachinidae]; Biomyia Schiner, 1868 of Biomya Rondani, 1856, n. syn. [Tachinidae]; Bombilius Dufour, 1833 of Bombylius Linnaeus, 1758, n. syn. [Bombyliidae]; Bombylosoma Loew, 1862 of Bombylisoma Rondani, 1856, n. syn. [Bombyliidae]; Brachipalpus Rondani, 1845 of Brachypalpus Macquart, 1834, n. syn. [Syrphidae]; Brachipalpus Rondani, 1863 of Palpibracus Rondani, 1863, n. syn. [Muscidae]; Brachistoma Rondani, 1856 of Brachystoma Meigen, 1822, n. syn. [Brachystomatidae]; Brachychaeta Brauer \& Bergenstamm, 1889 of Brachicheta Rondani, 1861, n. syn. [Tachinidae]; Brachyglossum Bigot, 1858 of Leopoldius Rondani, 1843, n. syn. [Conopidae]; Brachyneura Oken, 1844 of Brachineura Rondani, 1840, n. syn. [Cecidomyiidae]; Caelomya Rondani, 1866 of Fannia Robineau-Desvoidy, 1830, n. syn. [Fanniidae]; Caelomyia Rondani, 1877 of Fannia Robineau-Desvoidy, 1830, n. syn. [Fanniidae]; Caenosia Westwood, 1840 of Coenosia Meigen, 1826, n. syn. [Muscidae]; Campilomiza Rondani, 1840 of Campylomyza Meigen, 1818, n. syn. [Cecidomyiidae]; Campylochaeta Bezzi \& Stein, 1907 of Campylocheta Rondani, 1859, n. syn. [Tachinidae]; Caricoea Rondani, 1856 of Coenosia Meigen, 1826, n. syn. [Muscidae]; Carpomyia Loew, 1862 of Carpomya Rondani, 1856, n. syn. [Tephritidae]; Cassidemya Rondani, 1861 of Cassidaemyia Macquart, 1835, n. syn. [Rhinophoridae]; Ceratoxia Costa, 1866 of Otites Latreille, 1804, n. syn. [Ulidiidae]; Ceratoxys Rondani, 1861 of Otites Latreille, 1804, n. syn. [Ulidiidae]; Chaetogena Bezzi \& Stein, 1907 of Chetogena Rondani, 1856, n. syn. [Tachinidae]; Chamemyia Rondani, 1875 of Chamaemyia Meigen, 1803, n. syn. [Chamaemyiidae]; Chaetoptilia Bezzi \& Stein, 1907 of Chetoptilia Rondani, 1862, n. syn. [Tachinidae]; Chatolyga Bigot, 1892 of Carcelia Robineau-Desvoidy, $1830, \mathbf{n}$. syn. [Tachinidae]; Chersodromya Rondani, 1856 of Chersodromia Haliday, 1851, n. syn. [Hybotidae]; Chetilya Rondani, 1861 of Chetina Rondani, 1856, n. syn. [Tachinidae]; Chilopogon Bezzi, 1902 of Dasypogon Meigen, $1803, \mathbf{n}$. syn. [Asilidae]; Chiromya Agassiz, 1846 of Chyromya Robineau-Desvoidy, 1830, n. syn. [Chyromyidae]; Chlorisoma Rondani, 1861 of Microchrysa Loew, 1855, n. syn. [Stratiomyidae]; Chorthophila Rondani, 1856 of Phorbia RobineauDesvoidy, 1830, n. syn. [Anthomyiidae]; Chortofila Rondani, 1843 of Phorbia Robineau-Desvoidy, 1830, n. syn. [Anthomyiidae]; Chriorhyna Rondani, 1845 of Criorhina Meigen, 1822, n. syn. [Syrphidae]; Chrisogaster Rondani, 
1868 of Chrysogaster Meigen, 1803, n. syn. [Syrphidae]; Chryorhina Rondani, 1856 of Criorhina Meigen, 1822, n. syn. [Syrphidae]; Chryorhyna Rondani, 1857 of Criorhina Meigen, 1822, n. syn. [Syrphidae]; Chrysoclamys Rondani, 1856 of Ferdinandea Rondani, 1844, n. syn. [Syrphidae]; Chrysomya Rondani, 1856 of Microchrysa Loew, 1855, n. syn. [Stratiomyidae]; Chrysopila Rondani, 1844 of Chrysopilus Macquart, 1826, n. syn. [Rhagionidae]; Chyrosia Rondani, 1866 of Chirosia Rondani, 1856, n. syn. [Anthomyiidae]; Clytiomyia Rondani, 1862 of Clytiomya Rondani, 1861, n. syn. [Tachinidae]; Conopoejus Bigot, 1892 of Conops Linnaeus, 1758, n. syn. [Conopidae]; Criorhyna Rondani, 1865 of Criorhina Meigen, 1822, n. syn. [Syrphidae]; Criptopalpus Rondani, 1863 of Cryptopalpus Rondani, 1850, n. syn. [Tachinidae]; Crysogaster Rondani, 1865 of Chrysogaster Meigen, 1803, n. syn. [Syrphidae]; Crysops Rondani, 1844 of Chrysops Meigen, 1803, n. syn. [Tabanidae]; Cyrthoneura Rondani, 1863 of Graphomya Robineau-Desvoidy, 1830, $\mathbf{n}$. syn. [Muscidae]; Cyrthoplaeba Rondani, 1857 of Cyrtophloeba Rondani, 1856, n. syn. [Tachinidae]; Cyrthosia Rondani, 1863 of Cyrtosia Perris, 1839, n. syn. [Mythicomyiidae]; Cystogaster Walker, 1856 of Cistogaster Latreille, $1829, \mathbf{n}$. syn. [Tachinidae]; Cyterea Rondani, 1856 of Cytherea Fabricius, 1794, n. syn. [Bombyliidae]; Dactyliscus Bigot, 1857 of Habropogon Loew, 1847, n. syn. [Asilidae]; Dasiphora Rondani, 1856 of Dasyphora Robineau-Desvoidy, 1830, n. syn. [Muscidae]; Dasipogon Dufour, 1833 of Dasypogon Meigen, 1803, n. syn. [Asilidae]; Dasyneura Oken, 1844 of Dasineura Rondani, 1840, n. syn. [Cecidomyiidae]; Dexiomorpha Mik, 1887 of Estheria Robineau-Desvoidy, n. syn. [Tachinidae]; Dichaetophora Becker, 1905 of Dichetophora Rondani, 1868, n. syn. [Sciomyzidae]; Dicheta Rondani, 1856 of Dichaeta Meigen, 1830, n. syn. [Ephydridae]; Dictia Rondani, 1856 of Dictya Meigen, 1803, n. syn. [Sciomyzidae]; Dionea Rondani, 1861 of Dionaea Robineau-Desvoidy, 1830, n. syn. [Tachinidae]; Ditricha Rondani, 1871 of Dithryca Rondani, 1856, n. syn. [Tephritidae]; Dolicopeza Rondani, 1856 of Dolichopeza Meigen, 1830, n. syn. [Tipulidae]; Doricera Rondani, 1856 of Dorycera Meigen, 1830, n. syn. [Ulidiidae]; Drimeia Rondani, 1877 of Drymeia Meigen, 1826, n. syn. [Muscidae]; Drimeja Rondani, 1856 of Drymeia Meigen, 1826, n. syn. [Muscidae]; Driomyza Rondani, 1844 of Dryomyza Fallén, 1820, n. syn. [Dryomyzidae]; Driope Rondani, 1868 of Dryope Robineau-Desvoidy, 1830, n. syn. [Dryomyzidae]; Dryomiza Rondani, 1869 of Dryomyza Fallén, 1820, n. syn. [Dryomyzidae]; Dynera Rondani, 1861 of Dinera Robineau-Desvoidy, 1830, n. syn. [Tachinidae]; Dytricha Rondani, 1870 of Dithryca Rondani, 1856, n. syn. [Tephritidae]; Elachysoma Rye, 1881 of Elachisoma Rondani, 1880, n. syn. [Sphaeroceridae]; Elaeophila Marschall, 1873 of Eloeophila Rondani, 1856, n. syn. [Limoniidae]; Emerodromya Rondani, 1856 of Hemerodromia Meigen, 1822, n. syn. [Empididae]; Engyzops Bezzi \& Stein, 1907 of Eggisops Rondani, 1862, n. syn. [Calliphoridae]; Entomybia Rondani, 1879 of Braula Nitzsch, 1818, n. syn. [Braulidae]; Epidesmya Rondani, 1861 of Acidia RobineauDesvoidy, 1830, n. syn. [Tephritidae]; Erinnia Rondani, 1856 of Erynnia Robineau-Desvoidy, 1830, n. syn. [Tachinidae]; Eristalomyia Kittel \& Kreichbaumer, 1872 of Eristalomya Rondani, 1857, n. syn. [Syrphidae]; Esteria Rondani, 1862 of Estheria Robineau-Desvoidy, 1830, n. syn. [Tachinidae]; Exatoma Rondani, 1856 of Hexatoma Meigen, 1803, n. syn. [Tabanidae]; Exochila Mik, 1885 of Hammerschmidtia Schummel, 1834, n. syn. [Syrphidae]; Fisceria Rondani, 1856 of Fischeria Robineau-Desvoidy, 1830, n. syn. [Tachinidae]; Gedia Rondani, 1856 of Gaedia Meigen, 1838, n. syn. [Tachinidae]; Gimnocheta Rondani, 1859 of Gymnocheta Robineau-Desvoidy, 1830, n. syn. [Tachinidae]; Gimnosoma Rondani, 1862 of Gymnosoma Meigen, 1803, n. syn. [Tachinidae]; Gonirhinchus Lioy, 1864 of Myopa Fabricius, 1775, n. syn. [Conopidae]; Gonirhynchus Marschall, 1873 of Myopa Fabricius, 1775, n. syn. [Conopidae]; Gononeura Oldenberg, 1904 of Gonioneura Rondani, 1880, n. syn. [Sphaeroceridae]; Graphomia Rondani, 1862 of Graphomya Robineau-Desvoidy, 1830, n. syn. [Muscidae]; Gymnopha Rondani, 1856 of Mosillus Latreille, 1804, n. syn. [Ephydridae]; Hammobates Rondani, 1857 of Tachytrechus Haliday, 1851, n. syn. [Dolichopodidae]; Harrysia Rondani, 1865 of Lydina Robineau-Desvoidy, 1830, n. syn. [Tachinidae]; Hemathobia Rondani, 1862 of Haematobia Le Peletier \& Serville, 1828, n. syn. [Muscidae]; Hemerodromya Rondani, 1856 of Hemerodromia Meigen, 1822, n. syn. [Empididae]; Heryngia Rondani, 1857 of Heringia Rondani, 1856, n. syn. [Syrphidae]; Hidropota Lioy, 1864 of Hydrellia Robineau-Desvoidy, 1830, n. syn. [Ephydridae]; Hipostena Rondani, 1861 of Phyllomya Robineau-Desvoidy, 1830, n. syn. [Tachinidae]; Hirmophloeba Marschall, 1873 of Hyrmophlaeba Rondani, 1863, n. syn. [Nemestrinidae]; Histricia Rondani, 1863 of Hystricia Macquart, 1843, n. syn. [Tachinidae]; Hoemotobia Rondani, 1856 of Haematobia Le Peletier \& Serville, 1828, n. syn. [Muscidae]; Homalomya Rondani, 1866 of Fannia Robineau-Desvoidy, 1830, n. syn. [Fanniidae]; Homalostoma Bezzi \& Stein, 1907 of Billaea RobineauDesvoidy, 1830, n. syn. [Tachinidae]; Hoplisa Brauer \& Bergenstamm, 1889 of Oplisa Rondani, 1862, n. syn. [Rhinophoridae]; Hydrothaea Rondani, 1856 of Hydrotaea Robineau-Desvoidy, 1830, n. syn. [Muscidae]; Hylara Rondani, 1856 of Hilara Meigen, 1822, n. syn. [Empididae]; Hyrmoneura Rondani, 1863 of Hirmoneura Meigen, 1820 , n. syn. [Nemestrinidae]; Ilisomyia Osten Sacken, 1869 of Ormosia Rondani, 1856, n. syn. [Limoniidae]; Istochaeta Marschall, 1873 of Istocheta Rondani, 1859, n. syn. [Tachinidae]; Lamnea Rondani, 1861 of Erioptera Meigen, $1803, \mathbf{n}$. syn. [Limoniidae]; Lasiophthicus Rondani, 1856 of Scaeva Fabricius, 1805, n. syn. [Syrphidae]; Lestremya Rondani, 1856 of Lestremia Macquart, 1826, n. syn. [Cecidomyiidae]; Lidella De Galdo, 1856 of Lydella Robineau-Desvoidy, 1830, n. syn. [Tachinidae]; Lomacantha Lioy, 1864 of Lomachantha Rondani, 1859, n. syn. [Tachinidae]; Lomachanta Schiner, 1864 of Lomachantha Rondani, 1859, n. syn. [Tachinidae]; Loncoptera Rondani, 1856 of Lonchoptera Meigen, 1803, n. syn. [Lonchopteridae]; Lymnophora Blanchard, 1845 of Limnophora Robineau-Desvoidy, 1830, n. syn. [Muscidae]; Macherium Rondani, 1856 of Machaerium Haliday, 1832, n. syn. [Dolichopodidae]; Macrochaetum Bezzi, 1894 of Elachiptera Macquart, 1825, n. syn. [Chloropidae]; Macrochoetum Bezzi, 1892 of Elachiptera Macquart, 1825, 
n. syn. [Chloropidae]; Macroneura Rondani, 1856 of Diadocidia Ruthe, 1831, n. syn. [Diadocidiidae]; Marshamya Rondani, 1850 of Linnaemya Robineau-Desvoidy, 1830, n. syn. [Tachinidae]; Marsilia Bezzi \& Stein, 1907 of Tricoliga Rondani, 1859, n. syn. [Tachinidae]; Megachetum Rondani, 1856 of Dasyna Robineau-Desvoidy, 1830, n. syn. [Psilidae]; Megaloglossa Bezzi, 1907 of Platystoma Meigen, 1803, n. syn. [Platystomatidae]; Megera Rondani, 1859 of Senotainia Macquart, 1846, n. syn. [Sarcophagidae]; Melanomyia Rondani, 1868 of Melanomya Rondani, 1856, n. syn. [Calliphoridae]; Melizoneura Bezzi \& Stein, 1907 of Melisoneura Rondani, 1861, n. syn. [Tachinidae]; Mesomelaena Bezzi \& Stein, 1907 of Mesomelena Rondani, 1859, n. syn. [Sarcophagidae]; Micetina Rondani, 1861 of Mycetophila Meigen, 1803, n. syn. [Mycetophilidae]; Micetobia Rondani, 1861 of Mycetobia Meigen, 1818, n. syn. [Anisopodidae]; Micromyia Oken, 1844 of Micromya Rondani, 1840, n. syn. [Cecidomyiidae]; Miennis Rondani, 1869 of Myennis Robineau-Desvoidy, 1830, n. syn. [Ulidiidae]; Miopina Rondani, 1866 of Myopina Robineau-Desvoidy, 1830, n. syn. [Anthomyiidae]; Morjnia Rondani, 1862 of Morinia Robineau-Desvoidy, 1830, n. syn. [Calliphoridae]; Morphomyia Rondani, 1862 of Stomina Robineau-Desvoidy, 1830, n. syn. [Tachinidae]; Myatropa Rondani, 1857 of Myathropa Rondani, 1845, n. syn. [Syrphidae]; Mycetomiza Rondani, 1861 of Mycosia Rondani, 1861, n. syn. [Mycetophilidae]; Myiantha Rondani, 1877 of Fannia Robineau-Desvoidy, 1830, n. syn. [Fanniidae]; Myiathropa Rondani, 1868 of Myathropa Rondani, 1845, n. syn. [Syrphidae]; Myiocera Rondani, 1868 of Dinera Robineau-Desvoidy, 1830, n. syn. [Tachinidae]; Myiolepta Rondani, 1868 of Myolepta Newman, 1838, n. syn. [Syrphidae]; Myiospila Rondani, 1868 of Myospila Rondani, 1856, n. syn. [Muscidae]; Myltogramma Rondani, 1868 of Miltogramma Meigen, 1803, n. syn. [Sarcophagidae]; Myntho Rondani, 1845 of Mintho Robineau-Desvoidy, 1830, n. syn. [Tachinidae]; Myospyla Rondani, 1862 of Myospila Rondani, 1856, n. syn. [Muscidae]; Napoea Rondani, 1856 of Parydra Stenhammar, 1844, n. syn. [Ephydridae]; Neera Rondani, 1861 of Neaera Robineau-Desvoidy, 1830, n. syn. [Tachinidae]; Nemestrina Blanchard, 1845 of Nemestrinus Latreille, 1802, n. syn. [Nemestrinidae]; Nemorea Macquart, 1834 of Nemoraea RobineauDesvoidy, 1830, n. syn. [Tachinidae]; Nevrolyga Agassiz, 1846 of Neurolyga Rondani, 1840, n. syn. [Cecidomyiidae]; Nictia Rondani, 1862 of Nyctia Robineau-Desvoidy, 1830, n. syn. [Sarcophagidae]; Noteromyia Marschall, 1873 of Camilla Haliday, 1838, n. syn. [Camillidae]; Ociptera Rondani, 1862 of Cylindromyia Meigen, 1803, n. syn. [Tachinidae]; Onodonta Rondani, 1866 of Hydrotaea Robineau-Desvoidy, 1830, n. syn. [Muscidae]; Opegiocera Rondani, 1845 of Ancylorhynchus Berthold, 1827, n. syn. [Asilidae]; Ophira Rondani, 1844 of Hydrotaea RobineauDesvoidy, 1830, n. syn. [Muscidae]; Ornithoeca Kirby, 1880 of Ornithoica Rondani, 1878, n. syn. [Hippoboscidae]; Ornithomyia Macquart, 1835 of Ornithomya Latreille, 1804, n. syn. [Hippoboscidae]; Orthochile Blanchard, 1845 of Ortochile Latreille, 1809, n. syn. [Dolichopodidae]; Oxicera Rondani, 1856 of Oxycera Meigen, 1803, n. syn. [Stratiomyidae]; Oxina Rondani, 1856 of Oxyna Robineau-Desvoidy, 1830, n. syn. [Tephritidae]; Ozyrhinchus Rondani, 1861 of Ozirhincus Rondani, 1840, n. syn. [Cecidomyiidae]; Oxyrhyncus Rondani, 1856 of Ozirhincus Rondani, 1840, n. syn. [Cecidomyiidae]; Pachigaster Rondani, 1856 of Pachygaster Meigen, 1803, n. syn. [Stratiomyidae]; Pachimeria Rondani, 1856 of Pachymeria Stephens, 1829, n. syn. [Empididae]; Pachipalpus Rondani, 1856 of Cordyla Meigen, 1803, n. syn. [Mycetophilidae]; Pachirhyna Rondani, 1845 of Nephrotoma Meigen, 1803, n. syn. [Tipulidae]; Pachirina Rondani, 1840 of Nephrotoma Meigen, 1803, n. syn. [Tipulidae]; Pachistomus Rondani, 1856 of Xylophagus Meigen, 1803, n. syn. [Xylophagidae]; Pangonia Macquart, 1834 of Pangonius Latreille, 1802, n. syn. [Tabanidae]; Pentetria Rondani, 1856 of Penthetria Meigen, 1803, n. syn. [Bibionidae]; Perichaeta Herting, 1984 of Policheta Rondani, 1856, n. syn. [Tachinidae]; Perichoeta Bezzi, 1894 of Policheta Rondani, 1856, n. syn. [Tachinidae]; Phalacromyia Costa, 1866 of Copestylum Macquart, 1846, n. syn. [Syrphidae]; Phicodromia Rondani, 1866 of Malacomyia Westwood, 1840, n. syn. [Coelopidae]; Phillophaga Lioy, 1864 of Asphondylia Loew, 1850, n. syn. [Cecidomyiidae]; Phito Rondani, 1861 of Phyto Robineau-Desvoidy, 1830, n. syn. [Rhinophoridae]; Phitomyptera Lioy, 1864 of Phytomyptera Rondani, 1845, n. syn. [Tachinidae]; Phitophaga Lioy, 1864 of Cecidomyia Meigen, 1803, n. syn. [Cecidomyiidae]; Phloebotomus Rondani, 1856 of Phlebotomus Rondani \& Berté, 1840, n. syn. [Psychodidae]; Phorichaeta Brauer \& Bergenstamm, 1889 of Periscepsia Gistel, 1848, n. syn. [Tachinidae]; Phrino Rondani, 1861 of Phryno RobineauDesvoidy, 1830, n. syn. [Tachinidae]; Phrixe Rondani, 1862 of Phryxe Robineau-Desvoidy, 1830, n. syn. [Tachinidae]; Phthyria Rondani, 1856 of Phthiria Meigen, 1803, n. syn. [Bombyliidae]; Phtyria Rondani, 1863 of Phthiria Meigen, 1803, n. syn. [Bombyliidae]; Phyllodromya Rondani, 1856 of Phyllodromia Zetterstedt, 1837, n. syn. [Empididae]; Phytofaga Rondani, 1843 of Cecidomyia Meigen, 1803, n. syn. [Cecidomyiidae]; Phytomyzoptera Bezzi, 1906 of Phytomyptera Rondani, 1845, n. syn. [Tachinidae]; Platiparea Rondani, 1870 of Platyparea Loew, 1862, n. syn. [Tephritidae]; Platistoma Lioy, 1864 of Platystoma Meigen, 1803, n. syn. [Platystomatidae]; Platychyra Rondani, 1859 of Panzeria Robineau-Desvoidy, 1830, n. syn. [Tachinidae]; Platynochetus Rondani, 1845 of Platynochaetus Wiedemann, 1830, n. syn. [Syrphidae]; Polychaeta Schiner, 1868 of Policheta Rondani, 1856, n. syn. [Tachinidae]; Polycheta Schiner, 1861 of Policheta Rondani, 1856, n. syn. [Tachinidae]; Porrhocondyla Agassiz, 1846 of Porricondyla Rondani, 1840, n. syn. [Cecidomyiidae]; Porrycondyla Walker, 1874 of Porricondyla Rondani, 1840, n. syn. [Cecidomyiidae]; Prosopaea Brauer \& Bergenstamm, 1889 of Prosopea Rondani, 1861, n. syn. [Tachinidae]; Psicoda Rondani, 1840 of Psychoda Latreille, 1797, n. syn. [Psychodidae]; Psylopus Rondani, 1850 of Sciapus Zeller, 1842, n. syn. [Dolichopodidae]; Pteropectria Rondani, 1869 of Herina Robineau-Desvoidy, 1830, n. syn. [Ulidiidae]; Pterospylus Bigot, 1857 of Syneches Walker, 1852, n. syn. [Hybotidae]; Pticoptera Rondani, 1856 of Ptychoptera Meigen, 1803, n. syn. [Ptychopteridae]; Ptilocheta Rondani, 1857 of Zeuxia Meigen, 1826, n. syn. [Tachinidae]; 
Ptilochoeta Bezzi, 1894 of Zeuxia Meigen, 1826, n. syn. [Tachinidae]; Ptylocera Rondani, 1861 of Zeuxia Meigen, 1826, n. syn. [Tachinidae]; Ptylops Rondani, 1859 of Macquartia Robineau-Desvoidy, 1830, n. syn. [Tachinidae]; Pyragrura Rondani, 1861 of Labigastera Macquart, 1834, n. syn. [Tachinidae]; Pyrrhosia Bezzi \& Stein, 1907 of Leskia Robineau-Desvoidy, 1830, n. syn. [Tachinidae]; Ragio Scopoli, 1777 of Rhagio Fabricius, 1775, n. syn. [Rhagionidae]; Raimondia Rondani, 1879 of Raymondia Frauenfeld, 1855, n. syn. [Hippoboscidae]; Ramphina Rondani, 1856 of Rhamphina Macquart, 1835, n. syn. [Tachinidae]; Ramphomya Rondani, 1845 of Rhamphomyia Meigen, 1822, n. syn. [Empididae]; Raphium Latreille, 1829 of Rhaphium Meigen, 1803, n. syn. [Dolichopodidae]; Rhynchomyia Macquart, 1835 of Rhyncomya Robineau-Desvoidy, 1830, n. syn. [Rhiniidae]; Rhyncosia Rondani, 1861 of Aphria RobineauDesvoidy, 1830, n. syn. [Tachinidae]; Rhynophora Rondani, 1861 of Rhinophora Robineau-Desvoidy, 1830, n. syn. [Rhinophoridae]; Riphus Rondani, 1845 of Rhyphus Latreille, 1804, n. syn. [Anisopodidae]; Ripidia Rondani, 1856 of Rhipidia Meigen, 1818, n. syn. [Limoniidae]; Sarcopaga Rondani, 1856 of Sarcophaga Meigen, 1826, n. syn. [Sarcophagidae]; Scatomiza Rondani, 1866 of Scathophaga Meigen, 1803, n. syn. [Scathophagidae]; Schaenomyza Rondani, 1866 of Schoenomyza Haliday, 1833, n. syn. [Muscidae]; Sciomiza Rondani, 1856 of Sciomyza Fallén, $1820, \mathbf{n}$. syn. [Sciomyzidae]; Sciopila Rondani, 1856 of Sciophila Meigen, 1818, n. syn. [Mycetophilidae]; Serromya Rondani, 1856 of Serromyia Meigen, 1818, n. syn. [Ceratopogonidae]; Seseromyia Costa, 1866 of Cosmina Robineau-Desvoidy, 1830, n. syn. [Rhiniidae]; Sibistroma Rondani, 1856 of Sybistroma Meigen, 1824, n. syn. [Dolichopodidae]; Simplecta Rondani, 1856 of Symplecta Meigen, 1830, n. syn. [Limoniidae]; Sinapha Rondani, 1856 of Synapha Meigen, 1818, n. syn. [Mycetophilidae]; Siritta Rondani, 1844 of Syritta Le Peletier \& Serville, 1828, n. syn. [Syrphidae]; Somatolia Bezzi \& Stein, 1907 of Lydina Robineau-Desvoidy, 1830, n. syn. [Tachinidae]; Somomia Rondani, 1862 of Calliphora Robineau-Desvoidy, 1830, n. syn. [Calliphoridae]; Somomyia Rondani, 1868 of Calliphora Robineau-Desvoidy, 1830, n. syn. [Calliphoridae]; Sphixaea Rondani, 1856 of Milesia Latreille, 1804, n. syn. [Syrphidae]; Sphyxaea Rondani, 1856 of Milesia Latreille, 1804, n. syn. [Syrphidae]; Sphyxapata Bigot, 1881 of Senotainia Macquart, 1846, n. syn. [Sarcophagidae]; Sphyximorpha Rondani, 1856 of Sphiximorpha Rondani, 1850, n. syn. [Syrphidae]; Spilomya Rondani, 1857 of Spilomyia Meigen, 1803, n. Syn. [Syrphidae]; Spiximorpha Rondani, 1857 of Sphiximorpha Rondani, $1850, \mathbf{n}$. syn. [Syrphidae]; Spixosoma Rondani, 1857 of Conops Linnaeus, 1758, n. syn. [Conopidae]; Spylographa Rondani, 1871 of Trypeta Meigen, 1803, n. syn. [Tephritidae]; Stenopterix Millet de la Turtaudière, 1849 of Craterina Olfers, 1816, n. syn. [Hippoboscidae]; Stomorhyna Rondani, 1862 of Stomorhina Rondani, 1861, n. syn. [Rhiniidae]; Stomoxis Latreille, 1797 of Stomoxys Geoffroy, 1762, n. syn. [Muscidae]; Syphona Rondani, 1844 of Siphona Meigen, $1803, \mathbf{n}$. syn. [Tachinidae]; Tachidromya Rondani, 1856 of Tachydromia Meigen, 1803, n. syn. [Hybotidae]; Tachipeza Rondani, 1856 of Tachypeza Meigen, 1830, n. syn. [Hybotidae]; Tanipeza Rondani, 1850 of Tanypeza Fallén, 1820, n. syn. [Tanypezidae]; Teicomyza Rondani, 1856 of Teichomyza Macquart, 1835, n. syn. [Ephydridae]; Telaira Rondani, 1862 of Thelaira Robineau-Desvoidy, 1830, n. syn. [Tachinidae]; Teremya Rondani, 1875 of Lonchaea Fallén, 1820, n. syn. [Lonchaeidae]; Thecomya Rondani, 1848 of Thecomyia Perty, 1833, n. syn. [Sciomyzidae]; Thlypsigaster Marschall, 1873 of Amictus Wiedemann, 1817, n. syn. [Bombyliidae]; Thlypsomyza Rondani, 1863 of Amictus Wiedemann, 1817, n. syn. [Bombyliidae]; Thrichogena Bezzi, 1894 of Loewia Egger, 1856, n. syn. [Tachinidae]; Thricogena Rondani, 1859 of Loewia Egger, 1856, n. syn. [Tachinidae]; Thricophticus Rondani, 1866 of Thricops Rondani, 1856, n. syn. [Muscidae]; Thriptocheta Lioy, 1864 of Campichoeta Macquart, 1835, n. syn. [Diastatidae]; Thryptochoeta Bezzi, 1891 of Campichoeta Macquart, 1835, n. syn. [Diastatidae]; Thyreodonta Marschall, 1873 of Stratiomys Geoffroy, 1762 , n. syn. [Stratiomyidae]; Toxopora Rondani, 1856 of Toxophora Meigen, 1803, n. syn. [Bombyliidae]; Tricholiga Rondani, 1873 of Tricoliga Rondani, 1856, n. syn. [Tachinidae]; Trichophticus Rondani, 1871 of Thricops Rondani, 1856, n. syn. [Muscidae]; Tricocera Rondani, 1856 of Trichocera Meigen, 1803, n. syn. [Trichoceridae]; Tricolyga Schiner, 1861 of Tricoliga Rondani, 1856, n. syn. [Tachinidae]; Trigliphus Rondani, 1856 of Triglyphus Loew, 1840, n. syn. [Syrphidae]; Tripeta Rondani, 1856 of Trypeta Meigen, 1803, n. syn. [Tephritidae]; Triphera Rondani, 1861 of Tryphera Meigen, 1838, n. syn. [Tachinidae]; Triptocera Lioy, 1864 of Actia Robineau-Desvoidy, 1830, n. syn. [Tachinidae]; Tryptocera Macquart, 1844 of Actia Robineau-Desvoidy, 1830, n. syn. [Tachinidae]; Uromya Rondani, 1856 of Phania Meigen, 1824, n. syn. [Tachinidae]; Winthemya Rondani, 1859 of Winthemia Robineau-Desvoidy, 1830, n. syn. [Tachinidae]; Xiloteja Rondani, 1863 of Myolepta Newman, 1838, n. syn. [Syrphidae]; Xylomyia Marschall, 1873 of Xylomya Rondani, 1861, n. syn. [Xylomyidae]; Xyloteja Rondani, 1856 of Myolepta Newman, 1838, n. syn. [Syrphidae]; Xyphidicera Rondani, 1845 of Xiphidicera Macquart, 1834, n. syn. [Hybotidae]; Xyphocera Rondani, 1845 of Ancylorhynchus Berthold, 1827, n. syn. [Asilidae]; Zigoneura Rondani, 1840 of Zygoneura Meigen, 1830, n. syn. [Sciaridae]; Zophomya Rondani, 1859 of Zophomyia Macquart, 1835, n. syn. [Tachinidae]. Species-group namePsalida leucostoma Rondani, 1856 of Ocyptera simplex Fallén, 1815, n. syn. [Tachinidae].

Mycosia Rondani, 1861 is treated here as nomen dubium [Mycetophilidae]; Habropogon heteroneurus TimonDavid, 1951 is resurrected from junior synonymy with Asilus striatus Fabricius, 1794, new stat. [Asilidae].

Reversal of precedence is invoked for three cases of subjective synonymy to promote stability in nomenclature: Macquartia monticola Egger, 1856, nomen protectum and Proboscina longipes Rondani, 1856, nomen oblitum [in Tachinidae]; Loewia Egger, 1856, nomen protectum and Thrychogena Rondani, 1856, nomen oblitum [in Tachinidae]; Zygomyia Winnertz, 1863, nomen protectum and Bolithomyza Rondani, 1856, nomen oblitum [in Mycetophilidae]. 